ORIGINAL ARTICLE

\title{
Post-phototherapy neonatal bilirubin rebound: a potential cause of significant hyperbilirubinaemia
}

\author{
M Kaplan, E Kaplan, C Hammerman, N Algur, R Bromiker, M S Schimmel, \\ A I Eidelman

See end of article for authors' affiliations

Correspondence to: Dr M Kaplan, Department of Neonatology, Shaare Zedek Medical Center, PO Box 3235, Jerusalem 91031, Israel; kaplan@cc. huji.ac.il

Accepted

19 September 2005

Published Online First 13 October 2005
Aim: To determine the incidence of post-phototherapy neonatal plasma total bilirubin (PTB) rebound. Methods: A prospective clinical survey was performed on 226 term and near-term neonates treated with phototherapy in the well baby nursery of the Shaare Zedek Medical Center from January 2001 to September 2002. Neonates were tested for PTB 24 hours (between 12 and 36 hours) after discontinuation of phototherapy, with additional testing as clinically indicated. The main outcome measure, significant bilirubin rebound, was defined as a post-phototherapy PTB $\geqslant 256 \mu \mathrm{mol} / \mathrm{I}$. Phototherapy was not reinstituted in all cases of rebound, but rather according to clinical indications.

Results: A total of 30 (13.3\%) neonates developed significant rebound (mean (SD) PTB 287 (27) $\mu \mathrm{mol} / \mathrm{I}$, upper range $351 \mu \mathrm{mol} / \mathrm{l})$. Twenty two of these (73\%) were retreated with phototherapy at mean PTB 296 (29) $\mu \mathrm{mol} / \mathrm{l}$. Multiple logistic regression analysis showed significant risk for aetiological risk factors including positive direct Coombs test (odds ratio $2.44,95 \% \mathrm{Cl} 1.25$ to 4.74 ) and gestational age $<37$ weeks (odds ratio $3.21,95 \% \mathrm{Cl} 1.29$ to 7.96 ). A greater number of neonates rebounded among those in whom phototherapy was commenced $\leqslant 72$ hours $(26 / 152,17 \%)$ compared with $>72$ hours (4/74, 5.4\%) (odds ratio $3.61,95 \% \mathrm{Cl} 1.21$ to 10.77 ).

Conclusion: Post-phototherapy neonatal bilirubin rebound to clinically significant levels may occur, especially in cases of prematurity, direct Coombs test positivity, and those treated $\leqslant 72$ hours. These risk factors should be taken into account when planning post-phototherapy follow up.
B ecause the processes instrumental in the pathogenesis of the hyperbilirubinaemia may not yet have resolved by the time phototherapy is discontinued, post-phototherapy rebound may occur. However, data currently available are inadequate to formulate recommendations for or against post-phototherapy bilirubin testing. Many reports on the subject to date have been flawed by comprising retrospective chart reviews, analysis of rebound by determining the mean bilirubin value rather than peak post-treatment values, lack of preset definitions for rebound or indications for retreatment, pooling of data from term and extremely preterm neonates, or exclusion of neonates with haemolytic conditions from the analysis. ${ }^{1-6}$

The objectives of the study were: (1) to determine the incidence of post-phototherapy rebound to a predefined bilirubin concentration; and (2) to identify aetiological factors for hyperbilirubinaemia that could be used to select infants at risk for rebound.

\section{METHODS}

The study comprised an ongoing clinical survey of term and near-term neonates treated with phototherapy in the well baby nurseries of the Shaare Zedek Medical Center from January 2001 to September 2002. The Institutional Review Board of the institution gave approval for the study. Consecutively treated, otherwise healthy term and near-term infants ( $\geqslant 35$ weeks gestation) who had post-phototherapy plasma total bilirubin (PTB) determinations performed in our hospital's laboratory 24 hours (between 12 and 36 hours) after discontinuation of phototherapy, were included. Excluded from this analysis were those neonates who had been treated in our nursery but who did not return to our facilities for their post-bilirubin follow up.
Criteria for phototherapy for healthy term neonates were based on, but more stringent than, those of the 1994 American Academy of Pediatrics (AAP) Practice Parameter, ${ }^{7}$ as follows: <24 h: PTB $170 \mathrm{~mol} / \mathrm{l} ; 24-48 \mathrm{~h}: 205 \mathrm{~mol} / \mathrm{l}$; 49-72 h: $256 \mathrm{~mol} / \mathrm{l} ;>72 \mathrm{~h}: 29 \mathrm{l}-308 \mathrm{~mol} / \mathrm{l}$. In infants with risk factors, including gestational age $\leqslant 37$ weeks, direct Coombs positivity, glucose-6-phosphate dehydrogenase (G-6PD) deficiency, Down's syndrome, severe bruising, a strong family history of neonatal hyperbilirubinaemia, or evidence of infection, phototherapy was started at PTB concentrations 17-34 $\mu \mathrm{mol} / \mathrm{l}$ lower than described above.

Criteria for readmission for primary treatment of hyperbilirubinaemia included PTB concentrations $\geqslant 308-342 \mu \mathrm{mol} / \mathrm{l}$. Babies readmitted for primary or secondary (repeat) phototherapy were hospitalised in the same nursery in which they had been managed during their birth hospitalisation.

Neonates were routinely evaluated for the following aetiological entities for their hyperbilirubinaemia using clinical data or employing standard laboratory tests: direct Coombs positive $\mathrm{ABO}$ blood group heterospecificity or other blood group isoimmunisation, G-6-PD deficiency, direct Coombs negative $\mathrm{ABO}$ incompatibility, prematurity (gestational age $\leqslant 37$ weeks), and breast feeding. Less common entities were evaluated according to clinical indications.

Phototherapy was provided with babies lying supine in open bassinets, using overhead blue 4 lamp fluorescent units (Medela Phototherapy Lamp, Medela Medical Technology, Baar, Switzerland) placed about $25 \mathrm{~cm}$ above the baby. In many instances a phototherapy mattress was used as well (BiliBed, Medela Medical Technology, Baar, Switzerland). Light intensity, measured at the level of the skin of the baby's

Abbreviations: AAP, American Academy of Pediatrics; G-6-PD, glucose-6-phosphate dehydrogenase; PTB, plasma total bilirubin 
abdomen yielded a mean output of 17 (3) $\mu \mathrm{W} / \mathrm{cm}^{2} / \mathrm{nm}$ (Minolta Fluoro-lite meter 451, Japan). Breast feeding was encouraged and discontinued only in selected infants in whom the PTB continued to rise to $342 \mu \mathrm{mol} / \mathrm{l}$ or more despite phototherapy. During phototherapy PTB levels were determined at least twice daily, and more frequently if deemed necessary by clinical assessment. Phototherapy was continued until the serum total bilirubin concentration decreased to below $205 \mu \mathrm{mol} / \mathrm{l}$. In those cases of early hyperbilirubinaemia in whom PTB concentrations did not exceed $205 \mu \mathrm{mol} / \mathrm{l}$, treatment was discontinued when PTB concentrations stabilised and became lower than the 75th centile on the hour of life specific bilirubin nomogram. ${ }^{8}$

\section{Protocol for post-phototherapy rebound}

Nursery protocol includes a post-phototherapy PTB determination 24 hours (between 12 and 36 hours) after discontinuation of phototherapy, but does not require babies to remain hospitalised. Those neonates whose postphototherapy PTB concentration was lower than that at the time of discontinuation of phototherapy were discharged from follow up. Those in whom the repeat PTB was $\geqslant 120 \%$ of the post-phototherapy PTB, or $\geqslant 239 \mu \mathrm{mol} / \mathrm{l}$, were followed up at 12-24 hour intervals until either stabilisation/decrease of the PTB concentrations, or reinstitution of phototherapy. Phototherapy was recommenced at the discretion of the attending neonatologist, but not usually at PTB values $<256 \mu \mathrm{mol} / \mathrm{l}$. The decision to reinstitute phototherapy was influenced by the presence of risk factors for hyperbilirubinaemia.

\section{Data analysis}

Significant post-phototherapy rebound was arbitrarily defined, for the purpose of the study, as a PTB concentration that had risen to $\geqslant 256 \mu \mathrm{mol} / \mathrm{l}$ in a neonate who had been treated under phototherapy according to our protocol (see discussion).

The number of neonates with post-phototherapy PTB rebound $\geqslant 256 \mu \mathrm{mol} / \mathrm{l}$ was determined. The highest postphototherapy PTB was noted, although this was not always equivalent to the natural peak, depending on the reinstitution of phototherapy. The number of neonates retreated with phototherapy was recorded. The fact that a baby did develop rebound according to our definition, did not necessarily imply that that baby was retreated with phototherapy. To determine which aetiological groups were at significant risk for rebound hyperbilirubinaemia, data were further analysed by comparing rebound between aetiological conditions including direct Coombs positivity, prematurity, G-6-PD deficiency, $\mathrm{ABO}$ blood group incompatibility with negative direct Coombs test, and breast feeding as the only aetiology, using multiple logistic regression analysis. Early institution of phototherapy $(\leqslant 72 \mathrm{~h}$ ) was compared with those with later onset using odds ratios and 95\% confidence intervals.

Continuous variables were compared using Student's $t$ test and categorical variables were compared using $\chi^{2}$ analysis. Significance was defined as a p value $<0.05$ or, in the case of odds ratio determination, a 95\% confidence interval that did not include the digit 1 .

\section{Estimation of sample size}

Presuming equal numbers of neonates with haemolytic and non-haemolytic conditions, a $15 \%$ rate of rebound in the haemolytic group, and a $5 \%$ rate in the non-haemolytic group, with a power or 0.8 and an alpha value of 0.05 , it was estimated that a total of 160 patients would be necessary to compare babies with increased haemolysis with those who did not have haemolysis.

\section{Laboratory methods}

PTB was measured routinely in the hospital's clinical biochemistry laboratory by a direct spectrophotometric method using spun capillary tube samples (Wako Bilirubin Tester, Wako Pure Chemical Industries Ltd, Osaka, Japan).

Direct Coombs testing and G-6-PD screening were performed by routine laboratory methods.

\section{RESULTS}

A total of 312 neonates were treated with phototherapy during the study period. Of these, 226 (72\%) returned for follow up at our Medical Center and were thus eligible for inclusion. Mean (SD) values for birth weight and gestational age were 3204 (445) g and 39 (2) weeks, respectively. Two hundred and six (91\%) were born vaginally, and the male:female ratio was 134:92. Two hundred neonates $(88.5 \%)$ were either totally or partially breast feeding. Thirty of the $226(13 \%)$ were readmitted for primary phototherapy: demographic data did not differ significantly between those receiving primary phototherapy during their birth hospitalisation and those readmitted.

Of the 196 neonates who received primary phototherapy during their birth hospitalisation, $110(56 \%)$ had a positive direct Coombs test (106 were ABO heterospecific, three had anti-e and one anti-c isoimmunisation, and one required exchange transfusion). Forty three $(21.9 \%)$ were $\leqslant 37$ weeks gestation, 17 (8.7\%) were G-6-PD deficient, and 20 (10.2\%) were $\mathrm{ABO}$ heterospecific with a negative direct Coombs test (some overlap occurred between entities). In the subgroup of 30 neonates rehospitalised for primary phototherapy, all of whom were breast feeding, six $(20 \%)$ were $\leqslant 37$ gestational weeks, two $(6.6 \%)$ were direct Coombs positive, five (16.6\%) were G-6-PD deficient, and five (16.6\%) were ABO incompatible but with a negative direct Coombs test. Data regarding PTB values at onset and discontinuation of phototherapy, and the number of neonates with significant bilirubin rebound in both subgroups, are presented in table 1.

Overall, 30 neonates (13.2\%) had post-phototherapy PTB concentrations which rebounded to $\geqslant 256 \mu \mathrm{mol} / \mathrm{l}$. The mean (SD) PTB value of the rebounding neonates was 298 (29) $\mu \mathrm{mol} / \mathrm{l}$. The highest PTB concentration noted was $351 \mu \mathrm{mol} / \mathrm{l}$, while in three neonates rebound PTB value exceeded $342 \mu \mathrm{mol} / \mathrm{l}$. The number of neonates rebounding in each aetiological subgroup were compared using multiple logistic regression analysis: of all the aetiological subgroups studied, significance was shown only for those with a positive direct Coombs test (odds ratio $2.44,95 \%$ CI 1.25 to $4.74, \mathrm{p}=0.028$ ) and gestational age $<37$ weeks (odds ratio 3.21 , 95\% CI 1.29 to $7.96, p=0.036$ ). Furthermore, a greater number of neonates rebounded among those in whom phototherapy was commenced $\leqslant 72$ hours $(26 / 152,17 \%)$ compared with $>72$ hours $(4 / 74,5.4 \%$ ) (odds ratio $3.61,95 \%$ CI 1.21 to 10.77).

Of the 30 neonates with significant rebound, 22 (73\%) were retreated with phototherapy, of whom 15 were still hospitalised during birth hospitalisation and seven were readmitted. Of those babies who received secondary phototherapy, 16 were direct Coombs test positive, five were $\leqslant 37$ weeks gestation, and one was G-6-PD deficient. Secondary phototherapy was commenced 42 (26) hours after discontinuation of the initial phototherapy (two neonates were retreated with phototherapy $<24$ hours after first discontinuation). Mean PTB at onset of repeat phototherapy was 296 (29) $\mu \mathrm{mol} / \mathrm{l}$.

\section{DISCUSSION}

Few studies have systematically studied the phenomenon of post-phototherapy rebound..$^{1-6}$ Yetman and colleagues, ${ }^{1}$ pooling term as well as premature and low birth weight neonates, 


\begin{tabular}{|c|c|c|c|}
\hline & $\begin{array}{l}\text { Primary phototherapy } \\
\text { during birth hospitalisation } \\
\text { ( } n=196 \text { ) }\end{array}$ & $\begin{array}{l}\text { Primary } \\
\text { phototherapy } \\
\text { during readmission } \\
\text { ( } \mathrm{n}=30 \text { ) }\end{array}$ & Significance \\
\hline $\begin{array}{l}\text { Age at onset }(h)^{*} \\
\text { PTB at onset }\left(\mu \mathrm{mol} / \mathrm{l}^{*}\right. \\
\text { Age at discontinuation* } \\
\text { PTB at discontinuation }{ }^{*} \\
\text { Duration of phototherapy }(\mathrm{h})^{*} \\
\text { Number of neonates rebounding to PTB } \\
\geqslant 256 \mu \mathrm{mol} / / \text { I }\end{array}$ & $\begin{aligned} 53(29) \\
251(53) \\
96(25) \\
182(20) \\
43(23) \\
30(15.3 \%)\end{aligned}$ & $\begin{array}{l}122(38) \\
318(22) \\
152(40) \\
182(18) \\
30(9) \\
0\end{array}$ & $\begin{array}{l}p<0.001 \\
p<0.001 \\
p<0.001 \\
N S \\
p=0.002 \\
p=0.04\end{array}$ \\
\hline
\end{tabular}

found that mean bilirubin levels at the time of rebound testing were significantly lower than at discontinuation of phototherapy, but did not supply information regarding peak rebound bilirubin concentrations. Significant rebound was not defined by preset criteria, and only the first serum bilirubin determination performed within 30 hours of phototherapy was included in their analysis. Lazar and colleagues ${ }^{2}$ followed serum bilirubin levels to their natural peak in term neonates with non-haemolytic hyperbilirubinaemia. The upper range of rebound bilirubin reached a peak of $267 \mu \mathrm{mol} / \mathrm{l}$. However, the data from this series cannot be extrapolated to neonates with haemolytic conditions. Maisels and $\mathrm{Kring}^{3}$ used an episode of repeat phototherapy as an index of bilirubin rebound, although in this study preset criteria for repeat phototherapy were not set. Secondary phototherapy was instituted in $8.2 \%$ of those who received early treatment during their birth hospitalisation, compared with only $0.7 \%$ of those readmitted for primary phototherapy. In some cases the serum bilirubin did rise $>342 \mu \mathrm{mol} / \mathrm{l}$. AlSaedi $^{4}$ followed neonates for 8.3 (5.3) hours post-phototherapy and documented decrease in mean post-phototherapy bilirubin concentrations, although upper limits of the rebound were not provided. Erdeve and colleagues ${ }^{5}$ found that bilirubin concentrations did not increase significantly in 375 neonates followed for up to 12 hours after discontinuation of phototherapy, although $5.1 \%$ of these neonates did have repeat phototherapy. However, neither the upper limit of the rebound range nor the criteria for repeat phototherapy in that study were stated. Del Vecchio et al studied 48 neonates, of whom one 36 week gestation infant was retreated with phototherapy for a rebound bilirubin level of $17.0 \mathrm{mg} / \mathrm{dl} .^{6}$ Brown and colleagues ${ }^{9}$ found minimal increases in serum bilirubin concentrations in $<2000 \mathrm{~g}$ birth weight infants treated with prophylactic phototherapy, but in babies $>2000$ g birth weight, treated only if they actually developed hyperbilirubinaemia, STB values continued to decline once phototherapy was discontinued. Tan and colleagues ${ }^{10}$ found that only 7.89 per 1000 neonates required retreatment with phototherapy, although again, preset criteria were not established.

In contrast, our patients were treated according to preset guidelines. Significant rebound was strictly defined, and neonates were analysed according to aetiological subgroup. Neonates with haemolytic conditions were included, and upper limits of rebound bilirubin documented. Rebound occurred in $13.2 \%$ of neonates, and exceeded $342 \mu \mathrm{mol} / \mathrm{l}$, a level of bilirubin of concern in patients with haemolysis, in several cases. At highest risk were premature infants or those with a positive direct Coombs test. The increased risk associated with those in whom phototherapy was instituted within the first 72 hours of life probably reflects the higher number with active haemolysis within this subgroup. No subgroup was exempt from significant rebound except for those rehospitalised for primary phototherapy. The explanation for this may that few babies in the rehospitalisation group had haemolytic conditions, and, as a result of their older age, by the time phototherapy is discontinued, the natural postnatal rise in bilirubin will have levelled off, concomitant with maturation of the bilirubin conjugation process.

We pre-defined significant rebound as a PTB concentration of $\geqslant 256 \mu \mathrm{mol} / \mathrm{l}$ as this represented a $25 \%$ increase over our routine indication for discontinuation of phototherapy ( $205 \mu \mathrm{mol} / \mathrm{l}$ ) and is the beginning of the range of the 95th centile for bilirubin after the first 2-3 days of life. ${ }^{11}$ Our definition of rebound should not necessarily be equated with danger nor with a decision to reinstitute phototherapy. However, there were instances in which the rebound PTB value exceeded $342 \mu \mathrm{mol} / \mathrm{l}$, a bilirubin concentration which may be considered dangerous to actively haemolysing neonates or those $<37$ weeks gestation.

Because of the logistics involved in comprehensive follow up, and for accuracy and standardisation, we included in our analysis only those neonates tested for rebound in our hospital's laboratory. There was no reason to suspect that those babies who were followed up elsewhere were different from those included in this study. Our $72 \%$ retrieval rate does supply a reasonable reflection of the number of neonates affected by rebound and the drop out should not have resulted in substantial bias.

The 1994 Practice Parameter of the American Academy of Pediatrics (AAP), which was applicable at the time this study was being performed, but not applicable to neonates with haemolytic conditions, recommended that in most cases no further measurement of bilirubin is necessary following discontinuation of phototherapy, except possibly for cases in which phototherapy was instituted early and discontinued before the infant was 3-4 days old. ${ }^{1}$ The subsequent (2004) recommendations suggest that discharge from hospital need not be delayed in order to observe an infant for rebound. ${ }^{12}$ The AAP Subcommittee on Hyperbilirubinemia now recommends a follow up bilirubin measurement within 24 hours of discharge for those cases in which phototherapy was used for neonates with haemolytic diseases, initiated early, or discontinued before the infant is 3-4 days old. The Committee regards significant rebound among neonates who had been rehospitalised for hyperbilirubinaemia to be a rare occurrence and suggests a bilirubin measurement or clinical evaluation after 24 hours in these infants as a clinical option.

In light of our data, we recommend risk assessment in the selection of neonates to be tested for rebound hyperbilirubinaemia. Neonates with Coombs positive isoimmunisation, borderline prematurity, onset of phototherapy 


\section{What is already known on this topic}

- Post-phototherapy bilirubin rebound to bilirubin levels which may be of concern does occur

- The incidence of post-phototherapy rebound, defined prospectively, has not been adequately studied

\section{What this study adds}

- Neonates $<37$ weeks gestation, those with direct Coombs test positivity, and those treated $\leqslant 72$ hours, are at high risk for significant post-phototherapy rebound

$\leqslant 72$ hours, or a post-phototherapy rate of bilirubin rise greater than expected for age in hours, should be regarded as high risk. On the other hand, babies readmitted for primary phototherapy appear to be at low risk. No set of recommendations can be all-encompassing. Post-phototherapy bilirubin follow up should be incorporated using a combined approach of individualisation, evaluation of risk factors, and application of common sense.

\section{Authors' affiliations}

M Kaplan, C Hammerman, R Bromiker, M S Schimmel, A I Eidelman, Department of Neonatology, Shaare Zedek Medical Center, Jerusalem, Israel
N Algur, Clinical Biochemistry Laboratory, Shaare Zedek Medical Center, Jerusalem, Israel

Competing interests: none declared

\section{REFERENCES}

1 Yetman RJ, Parks DK, Huseby V, et al. Rebound bilirubin levels in infants receiving phototherapy. J Pediatr 1998;133:705-7.

2 Lazar L, Litwin A, Merlob P. Phototherapy for neonatal nonhemolytic hyperbilirubinemia. Clin Pediatr 1993;92:651-7.

3 Maisels MJ, Kring E. Rebound in serum bilirubin level following intensive phototherapy. Arch Pediatr Adolesc Med 2002;156:669-72.

4 Al-Saedi SA. Rebound hyperbilirubinemia in term infants after phototherapy. Saudi Med J 2002;23:1394-7.

5 Erdeve $\mathrm{O}$, Tiras $\mathrm{U}$, Dallar $\mathrm{Y}$. Rebound bilirubin measurement is not required for hyperbilirubinemia regardless of the background attributes of the newborns. J Trop Pediatr 2004;50:5.

6 Del Vecchio MT, Benstock MA, Sundel ER. Bilirubin rebound. J Pediatr 1999;135:531-2.

7 American Academy of Pediatrics, Provisional Committee for Quality Improvement and Subcommittee on Hyperbilirubinemia. Practice parameter: Management of hyperbilirubinemia in the healthy term newborn. Pediatrics 1994;94:558-65.

8 Bhutani VK, Johnson L, Sivieri EM. Predictive ability of a predischarge hour-specific serum bilirubin for subsequent significant hyperbilirubinemia in healthy term and near-term newborns. Pediatrics 1999; 103:6-14.

9 Brown AK, Kim MH, Wu PYK, et al. Efficacy of phototherapy in prevention and management of neonatal hyperbilirubinemia. Pediatrics 1985;75:393-400.

10 Tan KL, Lim GC, Boey KW. Efficacy of "high-intensity" blue-light and "standard" daylight phototherapy for non-haemolytic hyperbilirubinaemia. Acta Paediatr 1992;81:870-4.

11 Maisels MJ. The clinical approach to the jaundiced newborn. In: Maisels MJ, Watchko JF, eds. Neonatal jaundice. Amsterdam: Harwood Academic Publishers, 2000:139-68.

12 American Academy of Pediatrics, Subcommittee on Hyperbilirubinemia. Management of hyperbilirubinemia in the newborn infant 35 or more weeks of gestation. Pediatrics 2004;114:297-316.

\section{1 th European Forum on Quality Improvement in Health Care}

26-28 April 2006, Prague, Czech Republic

For further information please go to: www.quality.bmjpg.com

Book early to benefit from a discounted delegate rate 